\title{
Long-period quasi-periodic oscillations of a small-scale magnetic structure on the Sun
}

\author{
D. Y. Kolotkov ${ }^{1}$, V. V. Smirnova ${ }^{2,3}$, P. V. Strekalova ${ }^{3}$, A. Riehokainen ${ }^{2}$, and V. M. Nakariakov ${ }^{1,4}$ \\ ${ }^{1}$ Centre for Fusion, Space and Astrophysics, Department of Physics, University of Warwick, CV4 7AL, UK \\ e-mail: D.Kolotkov@warwick .ac.uk \\ 2 Tuorla Observatory and Department of Physics and Astronomy, University of Turku, 20500 Turku, Finland \\ 3 Central (Pulkovo) Astronomical Observatory, Pulkovskoe sh. 65, 196140 Saint-Petersburg, Russia \\ 4 St. Petersburg Branch, Special Astrophysical Observatory, Russian Academy of Sciences, 196140 St. Petersburg, Russia
}

Received 24 October 2016 / Accepted 22 December 2016

\begin{abstract}
Aims. Long-period quasi-periodic variations of the average magnetic field in a small-scale magnetic structure on the Sun are analysed. The structure is situated at the photospheric level and is involved in a facula formation in the chromosphere.

Methods. The observational signal obtained from the SDO/HMI line-of-sight magnetograms of the target structure has a nonstationary behaviour, and is therefore processed with the Hilbert-Huang Transform spectral technique.

Results. The empirical decomposition of the original signal and subsequent testing of the statistical significance of its intrinsic modes reveal the presence of the white and pink noisy components for the periods shorter and longer than 10 min, respectively, and a significant oscillatory mode. The oscillation is found to have a non-stationary period growing from approximately 80 to 230 min and an increasing relative amplitude, while the mean magnetic field in the oscillating structure is seen to decrease. The observed behaviour could be interpreted either by the dynamical interaction of the structure with the boundaries of supergranula cells in the region of interest or in terms of the vortex shedding appearing during the magnetic flux emergence.
\end{abstract}

Key words. Sun: faculae, plages - Sun: magnetic fields - Sun: oscillations

\section{Introduction}

Quasi-periodic modulations of solar signals on various spatial and temporal scales attract a growing interest among the research community (see e.g. Nakariakov et al. 2016, for the recent comprehensive review). Treating the magnetic field as a measurable parameter of the processes operating in the solar atmosphere (Bogdan 2000), these quasi-periodic variations are used as a diagnostic tool allowing for the estimation of the intrinsic plasma parameters and provide information about the dynamics and nature of the physical phenomena involved. Such quasi-periodicities are often detected in different solar structures, such as sunspots, plages and coronal loops (see e.g. Gelfreikh et al. 2006; Yuan et al. 2011; Abramov-Maximov et al. 2011; De Moortel \& Nakariakov 2012; Efremov et al. 2014). The typical periods of such oscillations range from a few seconds to several minutes (see e.g. Kupriyanova et al. 2010; Kobanov \& Chelpanov 2014; Sych 2016), while in some cases longer periodicities are also detected, for example in sunspots (Chorley et al. 2011; Bakunina et al. 2013; Abramov-Maximov et al. 2013). Particular attention was given to quasi-periodic fluctuations in low-atmospheric structures, and their leakage to higher levels of the solar atmosphere (Chorley et al. 2010; Smirnova et al. 2013).

In contrast, quasi-periodicities in smaller-scale solar magnetic structures with typical sizes of 4-10 arcsec, such as faculae and pores, are less studied as their direct observations were unavailable due to the insufficient resolution of the previously used ground-based and space-borne observational instruments. However, using the modern observational methods and newest satellite data with high spatial and temporal resolutions, now we are able to investigate the oscillatory behaviour of such smallscale structures in different layers of the solar atmosphere. For example, recently, Chelpanov et al. (2015) revealed such quasiperiodic oscillations in the facular magnetic knots with periods of approximately $3 \mathrm{~min}$. Additionally, the authors identified the longer-period oscillations (with periods of approximately 5-11 min) above the facula periphery regions in the $304 \AA$ spectral line. The detected spatial and temporal distributions of the oscillations were proposed by the authors to be prescribed by the magnetic field lines topology in facular regions. Similar periodicities and the corresponding dependence of the oscillation period upon the magnetic properties of a facular region were found in Kobanov et al. (2015). Freij et al. (2016) detected oscillations with periods from 3 to $20 \mathrm{~min}$ in the intensity of two isolated magnetic pores and interpreted them in terms of a slow magnetoacoustic sausage wave. Similarly, facular brightness was found to be dependent on the convective and wave motions in a facular region in Kostik \& Khomenko (2016). Periodicities in the 5-min range were detected. In addition to the solar atmosphere, Sun-like stars are also observed to exhibit quasi-periodic intensity fluctuations on various time scales, which can be attributed to the evolution of small-scale structures on their surfaces (see e.g. Karoff et al. 2013).

So far the longest detected periodicities in small-scale structures on the Sun are limited by a few tens of minutes and, in general, do not exceed a half an hour. In this Letter, we demonstrate the presence of a long-period oscillation with the period ranging from approximately 80 to $230 \mathrm{~min}$ in a long-living smallscale photospheric structure related to a facula formation visible 

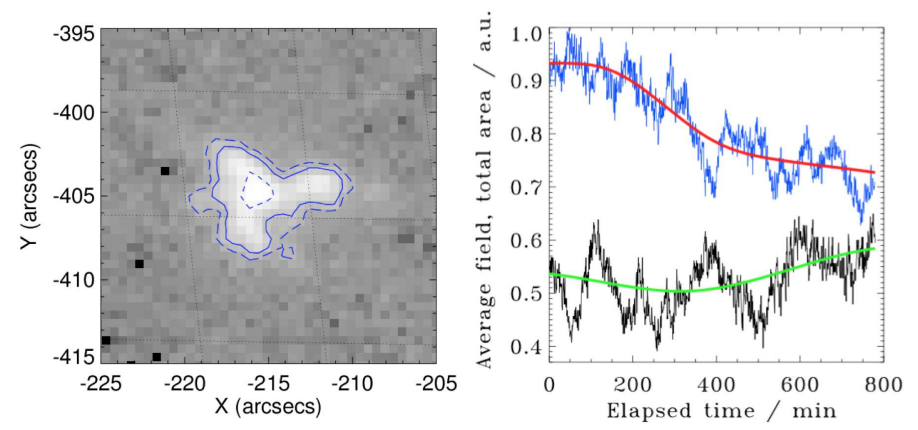

Fig. 1. Left: SDO/HMI line-of-sight magnetogram of the analysed magnetic structure, measured at 23:00:45 UT on the 6th July 2013. Blue contours show the magnetic field intensity levels of 70 (dash), 100 (solid) and 600 (dash) G. Right: temporal variations of the average lineof-sight component of the magnetic field (blue) in the structure, integrated over the whole $100 \mathrm{G}$ contour. The red solid line shows the longterm trend of the blue signal, determined as the last empirical mode with EMD (see Sect. 3). Variations of the total area inside the $100 \mathrm{G}$ contour are shown by the black solid line, with the long-term trend (green line) determined also with EMD. Both original time-series were normalised to their maximum values (shown in arbitrary units), and the total area signal was slightly shifted downwards for a better visualisation. The elapsed time starts at 23:00:45 UT on the 6th July 2013 and spans $13 \mathrm{~h}$ of observations with a cadence of $45 \mathrm{~s}$.

in the chromosphere. We analyse the time-series of the average line-of-sight component of the magnetic field obtained with SDO/HMI with the duration of $13 \mathrm{~h}$ using the Hilbert-Huang transform (HHT) technique (Huang et al. 1998; Huang \& Wu 2008). Due to its advantages in processing non-linear and nonstationary signals, this method is successfully used for the detection of quasi-periodicities in solar signals of various types (see e.g. Lee et al. 2015; Deng et al. 2015; Qu et al. 2015; Kolotkov et al. 2015; Käpylä et al. 2016; Xiang \& Qu 2016, for the recent results). Testing the significance of the identified empirical modes with the approach developed in Wu \& Huang (2004) and Kolotkov et al. (2016), we managed to distinguish the white and pink noisy components superimposed and a single significant oscillatory mode.

\section{Observations}

We used the line-of-sight magnetograms of the analysed smallscale structure, observed by the Solar Dynamics Observatory Helioseismic and Magnetic Imager instrument (SDO/HMI, Schou \& Larson 2011; Scherrer et al. 2012; Couvidat et al. 2016). The observations start at 23:00:45 UT on the 6th July 2013 and span $13 \mathrm{~h}$ with a cadence time of $45 \mathrm{~s}$. We note, that the spatial resolution of HMI ( 1 arcsec) allows us to successfully resolve the target structure of approximately 4-10 arcsec in size.

A snapshot of the analysed structure with the helioprojective Cartesian coordinates of its geometrical centre, $X$ and $Y$ being of approximately $[-216,-405]$ is shown in Fig. 1, left-hand panel at 23:00:45 UT. It is a positive polarity magnetic field structure, with the initial linear sizes of approximately $7 \times 5$ arcsec, estimated at the $100 \mathrm{G}$ level of intensity. During the observational interval, the position of the structure's geometrical centre experienced stochastic motions with the amplitudes being up to a typical size of the inter-granular lanes in the region of interest. In turn, the total area of the structure, estimated in the $100 \mathrm{G}$ intensity contour, varied quasi-periodically in time with a gradually increasing long-term trend (see the right-hand panel in Fig. 1).
We chose the contour of $100 \mathrm{G}$ as the most suitable for the analysis of the oscillations of the target structure as a whole. Indeed, it matches well the whole interior of the structure. On the other hand, its level is four times higher than that of the quiet Sun in the magnetogram, which is approximately $20-25 \mathrm{G}$. Hence, the possible oscillations of the analysed structure should not be affected by the background processes operating in the neighbouring quiet sun atmosphere. Right-hand panel of Fig. 1 also shows the temporal variations of the average magnetic field strength, obtained by the integration over the whole $100 \mathrm{G}$ contour, for each separate magnetogram during the whole observational interval of $13 \mathrm{~h}$. This time-series demonstrates a clear quasi-periodic behaviour of the average magnetic field in the structure and is a subject for a further spectral analysis.

\section{HHT spectral analysis and significance test}

Magnitude of the average magnetic field in the structure (see Fig. 1), obtained in the $100 \mathrm{G}$ contour, is processed with the Hilbert-Huang transform (HHT) technique (Huang et al. 1998). In contrast to a standard Fourier transform and wavelets, which are restricted by an a priori assignment of harmonic basis functions, HHT uses the empirical mode decomposition (EMD) of the signal of interest, iteratively searching for the local time scales naturally appearing in the signal, and expanding it into the basis derived directly from the data. Hence, due to its adaptive nature, EMD is essentially suitable for analysing non-stationary and non-linear variations.

Application of the EMD technique to the average magnetic field time-series shown in the right-hand panel of Fig. 1 allows us to extract 11 intrinsic empirical modes, with the aperiodic 11th mode being a long-term trend shown in Fig. 1. The detected modes 1-7 and 9-10 have nearly stable periods, while the apparent non-stationarity of the original signal is mainly attributed to mode 8 . Mean periods of the modes, estimated as $P=2 N / n$ where $N$ is the total length of the analysed signal and $n$ is the number of extrema in a mode, are shown in Table 1. Except the highest amplitude mode 8 (see the ratio of the modal standard deviation, $\sigma_{m}$ to the original signal standard deviation, $\sigma_{0}$ in Table 1), it shows the approximate dyadic behaviour of intrinsic modes 1-7 and 9-10, that is, their mean periods constitute a nearly doubling sequence, with each next period being approximately two times longer than the previous. The latter fact is typical for EMD when it operates with the signals containing white and coloured noisy components with a power law in their spectral energy distributions, and may indicate a random nature of these empirical modes (Flandrin et al. 2004). Similarly, the instant amplitudes of modes 1-7 and 9-10 were found to be normally distributed, which is also consistent with their random origin (Wu \& Huang 2004), in contrast to the oscillation revealed by mode 8 .

Writing the power spectral density $S$ as a function of the frequency $f$ as $S=1 / f^{\alpha}$, and following the recipes proposed by Wu \& Huang (2004) and Kolotkov et al. (2016), we are able to check the significance of the intrinsic modes, comparing them with the white and coloured noises, and empirically estimate the corresponding values of the power law index $\alpha$. Introducing the energy $E$ of an intrinsic mode as a sum of squares of its instant amplitudes in the time-series, Kolotkov et al. (2016) recently showed that for the intrinsic modes obtained from synthetic coloured noise samples, it is connected with the mean modal period $P$ as $E P^{1-\alpha}=$ const. The left-hand panel of Fig. 2 shows the modal energy-mean period dependence plotted in a logarithmic scale for all intrinsic modes detected in the current 
Table 1. Mean periods and ratios of the modal standard deviation, $\sigma_{m}$ to the original signal standard deviation, $\sigma_{0}$ of the empirical modes detected with EMD in the average magnetic field signal shown in Fig. 1, right-hand panel.

\begin{tabular}{lccccc}
\hline \hline & Period $(\min )$ & $\sigma_{m} / \sigma_{0}$ & & Period $(\min )$ & $\sigma_{m} / \sigma_{0}$ \\
\hline M1 & 2.3 & 0.13 & M6 & 36.3 & 0.14 \\
M2 & 4.0 & 0.09 & M7 & 74.3 & 0.14 \\
M3 & 6.4 & 0.07 & M8 & 120.0 & 0.31 \\
M4 & 10.6 & 0.07 & M9 & 156.0 & 0.08 \\
M5 & 17.9 & 0.07 & M10 & 312.0 & 0.10 \\
\hline
\end{tabular}
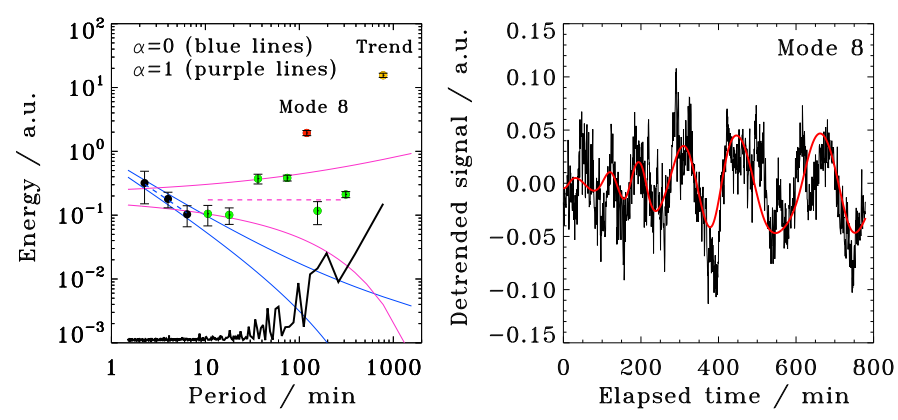

Fig. 2. Left: mean modal energy-period dependence plotted in a logarithmic scale, for all intrinsic modes detected with EMD in the signal of interest shown in the right-hand panel of Fig. 1 in blue. Values of the mean energies and periods of modes $1-3$ are shown by black circles while modes 4-7 and 9-10 are shown in green. The red circle indicates the mean energy and period of mode 8 shown in the right-hand panel. The yellow circle corresponds to the long-term trend of the signal, shown in the right-hand panel of Fig. 1 in red. Dashed blue and purple lines show the power law dependences $E P^{1-\alpha}=$ const., with the spectral slopes $\alpha=0$ and $\alpha=1$, respectively. Blue solid lines show the $99 \%$ and $1 \%$ confidence intervals of the white noise $(\alpha \approx 0)$, and the corresponding pink noise $(\alpha \approx 1)$ confidence intervals are shown by the purple solid lines. The black solid line shows the Fourier energy spectrum of the original signal shown in the right-hand panel of Fig. 1 in blue, shifted downwards by an appropriate normalisation for a better visualisation. Right: the detrended average magnetic field strength (black solid line) shown in the right-hand panel of Fig. 1 in blue, and its intrinsic mode 8 (red solid line) detected with EMD.

analysis. Modal energies are shown with the corresponding uncertainties, allowing the instant energies of each mode to vary within $2 \sigma$, where $\sigma$ is the half-level width of the instant energy distribution of each mode. Modes 1-3 (black circles) and modes 4-7 and 9-10 (green circles) are seen to lie around straight lines with the spectral slopes $\alpha$ of approximately 0 and 1, respectively. Here, these values of $\alpha$ are taken as illustrative examples only.

Figure 2 also illustrates the upper $(99 \%)$ and lower $(1 \%)$ confidence intervals of the white $(\alpha \approx 0)$ and pink $(\alpha \approx 1)$ noises, calculated in the assumption of a chi-squared modal energy distribution written in the form of Eq. (5) in Kolotkov et al. (2016). The applicability of the chi-squared law for both white and coloured noises was justified by Wu \& Huang (2004) and Kolotkov et al. (2016), respectively. The performed test shows that the detected shorter-period modes (1-3) have the energies lying strictly within the white noise confidence interval, that most likely indicates their white noise-like nature. In turn, the longer-period modes (4-7 and 9-10) have the energies lying within the pink noise confidence interval, and, hence, should be attributed to flickering random processes in the solar atmosphere. In contrast, energy of mode 8 is well above the corresponding confidence intervals of both white and pink noises, indicating the statistical significance of only that mode among the whole set of the intrinsic modes detected in the average magnetic field time-series (Fig. 1). The Fourier spectrum shown in Fig. 2 also demonstrates the change in the spectrum slope gradient, that is, in the noise colour, with a breaking point near the 20-min period, consistent with the results obtained with EMD. On the other hand, it does not have a pronounced periodic component. This discrepancy illustrates the advantage of EMD in the processing of non-stationary signals.

The right-hand panel of Fig. 2 demonstrates the normalised original signal with the long-term trend subtracted, and mode 8 . The mode has a clear quasi-periodic behaviour with a growing period, accompanied by a simultaneous increase in the amplitude. The empirical dependence of the instantaneous oscillation period upon the instantaneous oscillation amplitude of mode 8 is calculated with the use of the Hilbert transform, and is shown in Fig. 3, top left panel. It is a growing dependence that can be fitted by the power law of the form Period [min] = $a \times[\text { Amplitude } / \max (\text { Amplitude })]^{b}+c$, with $a \approx$ (141.56 \pm $56.91) \times 10^{3} \mathrm{~min}, b \approx 2.31 \pm 0.14$, and $c \approx 78.36 \pm 2.45 \mathrm{~min}$. According to this fit, the period of this mode increases from approximately $80 \mathrm{~min}$ (for the lowest amplitude) up to approximately $230 \mathrm{~min}$ (for the highest amplitude). The dependence of the instantaneous period of mode 8 upon the variation of the instant average magnetic field (the red curve in Fig. 1), shown in the top right panel of Fig. 3, is approximated by a power-law with the index of $-3 / 2$. Figure 3 also shows the dependences between the instantaneous period of mode 8 and the total area of the magnetic structure (bottom right panel) and its magnetic flux (bottom left panel), projected onto the plane of sky and determined as the product of the average magnetic field and the total area of the structure. No obvious correlation between the modal period and these two parameters was detected.

\section{Discussion and conclusions}

We analysed the quasi-periodic behaviour of the average magnetic field strength (shown in Fig. 1) measured with SDO/HMI in a photospheric small-scale magnetic element forming a facula at higher levels of the solar atmosphere. The spectral analysis performed with the HHT technique decomposed the signal into 11 intrinsic empirical modes, with the last (11th) mode being a long-term aperiodic trend of the signal. The significance test showed that the other nine empirical modes exhibit a nearly dyadic behaviour and are most likely related to the noisy components superimposed onto the original signal. They can be characterised by a power law index of approximately zero (thus representing the white noise component dominating in the shortperiod (2-6 min) part of the spectrum) and of approximately one (i.e. the pink or the so-called "flicker" noise dominating at longer periods of 10-310 min). Only a single mode was found to be significant. It has a gradually increasing oscillation amplitude, and its oscillation period grows with the amplitude from approximately 80 to $230 \mathrm{~min}$.

The detected white and coloured noises could manifest different physical processes of a natural or instrumental origin. The presence of the power-law-like regions in power spectra of the signals detected at various heights of the solar atmosphere seems to be a common feature. For example, Kolotkov et al. (2016) recently found similar values of the power law index, approximately $0.8-0.9$ in the extreme ultraviolet emission intensity variations observed with SDO/AIA in the upper photospheric (1600 $\AA$ ) layer of the non-flaring solar atmosphere. The higher, 

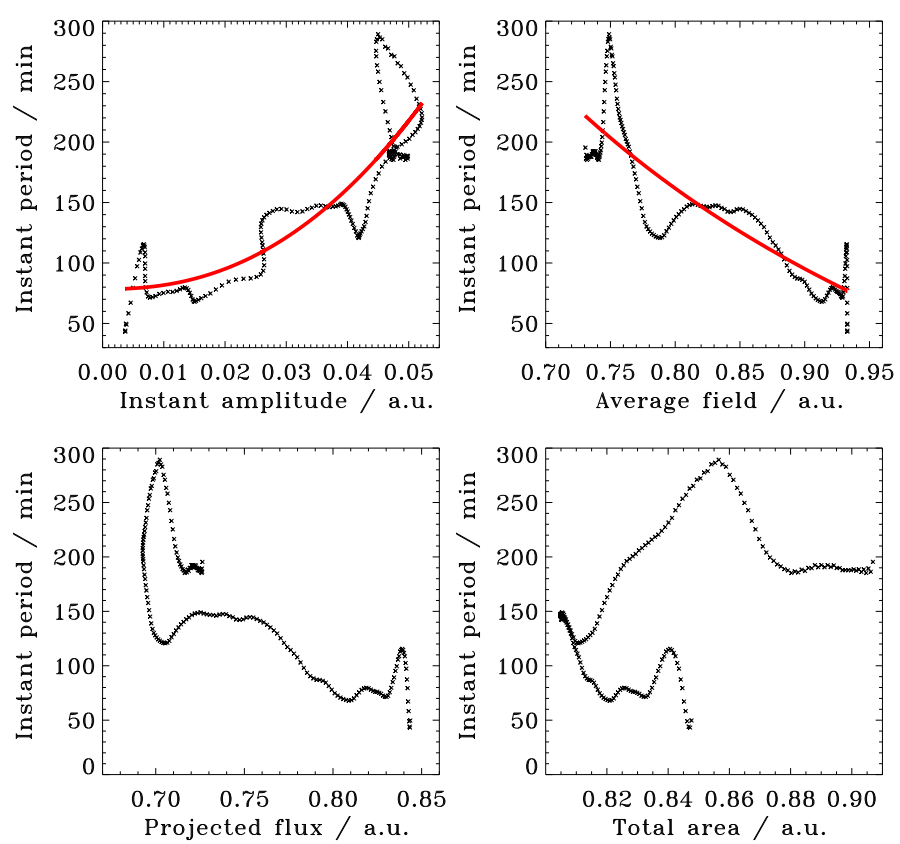

Fig. 3. Top left: dependence of the instantaneous oscillation period upon the instantaneous oscillation amplitude in mode 8 shown in the righthand panel of Fig. 2. The red solid line indicates the best fit of the empirical period-amplitude dependence by a power-law function (see Sect. 3 for the fitting details). Top right: dependence of the instantaneous period of mode 8 upon the long-term trend of the average magnetic field signal, shown in the right-hand panel of Fig. 1. The red curve shows the best fit of the empirical dependence by the power law function with the index of $-3 / 2$. Bottom left: dependence of the instantaneous period of mode 8 upon the long-term variation of the normalised line-of-sight magnetic flux of the structure, projected onto the plane of sky. Bottom right: dependence of the instantaneous period of mode 8 upon the longterm trend of the total area signal, shown in the right-hand panel of Fig. 1

chromospheric and coronal layers of the non-flaring solar atmosphere also often show the presence of randomly distributed processes characterised by the power law indices ranging from 1.2 to 2.3 depending upon the magnetic properties of the analysed region (see e.g. Ireland et al. 2015; Kolotkov et al. 2016).

The period of the statistically significant oscillation revealed by mode 8 , which is in the range from 80 to $230 \mathrm{~min}$, is far too long to be linked with a magnetohydrodynamic oscillation of the observed chromospheric or photospheric structure. Indeed, taking the Alfvén speed equal to the photospheric sound speed, $10 \mathrm{~km} \mathrm{~s}^{-1}$, we see that the natural magnetohydrodynamic oscillation with a 180 -min $(\approx 10000 \mathrm{~s})$ period should have a wavelength of approximately $100 \mathrm{Mm}$. On the other hand, similar periodicities could be induced by the dynamical interaction of photospheric magnetic structures with the boundaries of supergranula cells (Strekalova et al. 2016), however this mechanism requires further investigation. In particular, it is not clear whether the detected variation of the oscillation period with the amplitude and the mean magnetic field could be consistent with the motions caused by the supergranulation flows.

Another possibility is connected with the periodic motions appearing during the magnetic flux emergence (see e.g. Emonet et al. 2001; Cheung et al. 2006). In this effect, the period $P$ is prescribed by the vortex shedding, $P \propto d / V$, where $d$ is the emerging magnetic flux tube diameter and $V$ is the emergence speed (see e.g. Nakariakov et al. 2009). Taking that the flux tube diameter depends on the magnetic field strength as $B^{-1 / 2}$ (the magnetic flux conservation) and the emergence speed is proportional to the magnetic field, we obtain that the oscillation period scales with the magnetic field as $P \propto B^{-3 / 2}$. This behaviour is consistent with the scaling seen in Fig. 3. However, more detailed modelling of this phenomenon is required. The scaling of the instant oscillation period with the projected magnetic flux and the total photospheric area of the magnetic structure did not give conclusive results.

Acknowledgements. D.Y.K. acknowledges the Warwick Chancellor's International Scholarship. The work of V.V.S. was supported by the Russian Scientific Foundation grant No. 16-12-10448. V.V.S. also acknowledges CIMO (Centre of International Mobility) organisation at the Ministry of Education in Finland. grant TM-15-9794. V.M.N. acknowledges the European Research Council Research Project No. 321141 "SeismoSun".

\section{References}

Abramov-Maximov, V. E., Gelfreikh, G. B., Kobanov, N. I., Shibasaki, K., \& Chupin, S. A. 2011, Sol. Phys., 270, 175

Abramov-Maximov, V. E., Efremov, V. I., Parfinenko, L. D., Solov'ev, A. A., \& Shibasaki, K. 2013, PASJ, 65, S12

Bakunina, I. A., Abramov-Maximov, V. E., Nakariakov, V. M., et al. 2013, PASJ, $65, \mathrm{~S} 13$

Bogdan, T. J. 2000, Sol. Phys., 192, 373

Chelpanov, A. A., Kobanov, N. I., \& Kolobov, D. Y. 2015, Astron. Rep., 59, 968

Cheung, M. C. M., Moreno-Insertis, F., \& Schüssler, M. 2006, A\&A, 451, 303

Chorley, N., Hnat, B., Nakariakov, V. M., Inglis, A. R., \& Bakunina, I. A. 2010, A\&A, 513, A27

Chorley, N., Foullon, C., Hnat, B., Nakariakov, V. M., \& Shibasaki, K. 2011, A\&A, 529, A123

Couvidat, S., Schou, J., Hoeksema, J. T., et al. 2016, Sol. Phys., 291, 1887

De Moortel, I., \& Nakariakov, V. M. 2012, Phil. Trans. Roy. Soc. London Ser. A, 370,3193

Deng, L. H., Li, B., Xiang, Y. Y., \& Dun, G. T. 2015, J. Atmos. Sol.-Terr. Phys., 122,18

Efremov, V. I., Parfinenko, L. D., Solov'ev, A. A., \& Kirichek, E. A. 2014, Sol. Phys., 289, 1983

Emonet, T., Moreno-Insertis, F., \& Rast, M. P. 2001, ApJ, 549, 1212

Flandrin, P., Rilling, G., \& Goncalves, P. 2004, IEEE Sig. Process. Lett., 11, 112

Freij, N., Dorotovič, I., Morton, R. J., et al. 2016, ApJ, 817, 44

Gelfreikh, G. B., Nagovitsyn, Y. A., \& Nagovitsyna, E. Y. 2006, PASJ, 58,29

Huang, N. E., \& Wu, Z. 2008, Rev. Geophys., 46, RG2006

Huang, N. E., Shen, Z., Long, S. R., et al. 1998, Proc. Roy. Soc. London Ser. A, 454, 903

Ireland, J., McAteer, R. T. J., \& Inglis, A. R. 2015, ApJ, 798, 1

Käpylä, M. J., Käpylä, P. J., Olspert, N., et al. 2016, A\&A, 589, A56

Karoff, C., Campante, T. L., Ballot, J., et al. 2013, ApJ, 767, 34

Kobanov, N. I., \& Chelpanov, A. A. 2014, Astro. Rep., 58, 272

Kobanov, N., Kolobov, D., \& Chelpanov, A. 2015, Sol. Phys., 290, 363

Kolotkov, D. Y., Nakariakov, V. M., Kupriyanova, E. G., Ratcliffe, H., \& Shibasaki, K. 2015, A\&A, 574, A53

Kolotkov, D. Y., Anfinogentov, S. A., \& Nakariakov, V. M. 2016, A\&A, 592, A153

Kostik, R., \& Khomenko, E. 2016, A\&A, 589, A6

Kupriyanova, E. G., Melnikov, V. F., Nakariakov, V. M., \& Shibasaki, K. 2010, Sol. Phys., 267, 329

Lee, J. N., Cahalan, R. F., \& Wu, D. L. 2015, J. Atmos. Sol.-Terr. Phys., 132, 64 Nakariakov, V. M., Aschwanden, M. J., \& van Doorsselaere, T. 2009, A\&A, 502, 661

Nakariakov, V. M., Pilipenko, V., Heilig, B., et al. 2016, Space Sci. Rev., 200, 75 Qu, Z.-N., Feng, W., \& Liang, H.-F. 2015, RA\&A, 15, 879

Scherrer, P. H., Schou, J., Bush, R. I., et al. 2012, Sol. Phys., 275, 207

Schou, J., \& Larson, T. P. 2011, in AAS/Solar Physics Division Abstracts \#42, 1605

Smirnova, V., Riehokainen, A., Solov'ev, A., et al. 2013, A\&A, 552, A23

Strekalova, P., Nagovitsyn, Y. A., Riehokainen, A., \& Smirnova, V. 2016, Geomag. Aeron., 56, 1

Sych, R. 2016, AGU, Geophysical Monograph Series (Washington DC), 216, 467

Wu, Z., \& Huang, N. E. 2004, Proc. Roy. Soc. London Ser. A, 460, 1597

Xiang, N. B., \& Qu, Z. N. 2016, AJ, 151, 76

Yuan, D., Nakariakov, V. M., Chorley, N., \& Foullon, C. 2011, A\&A, 533, A116 